\title{
Existence of Strong Solutions for Nonlinear Systems of PDEs Arising in Convective Flow
}

\author{
Khaled Bouazzaoui $\mathbb{D},{ }^{1}$ Mohammed Aiboudi, ${ }^{1}$ and Sameh Elsayed Ahmed $\mathbb{D}^{2}$ \\ ${ }^{1}$ Département de Mathématiques, Laboratoire d'Analyse Mathématiques et Applications (LAMA), \\ Faculté des Sciences Exactes et Appliquées, Université Oran 1 Ahmed Ben Bella, Oran, Algeria \\ ${ }^{2}$ Department of Mathematics, Faculty of Sciences, South Valley University Qena, Qena 83523, Egypt \\ Correspondence should be addressed to Khaled Bouazzaoui; bouazzaoui_khaled@yahoo.com
}

Received 12 September 2021; Revised 22 November 2021; Accepted 2 December 2021; Published 10 January 2022

Academic Editor: Sining Zheng

Copyright (C) 2022 Khaled Bouazzaoui et al. This is an open access article distributed under the Creative Commons Attribution License, which permits unrestricted use, distribution, and reproduction in any medium, provided the original work is properly cited.

In this paper, we will study the existence of strong solutions for a nonlinear system of partial differential equations arising in convective flow, modeling a phenomenon of mixed convection created by a heated and diving plate in a porous medium saturated with a fluid. The main tools are Schäfer's fixed-point theorem, the Fredholm alternative, and some theorems on second-order elliptic operators.

\section{Introduction}

In recent years, many authors have studied the case of the semi-infinite vertical plane plate immersed in a porous medium saturated with a fluid. The following problem is derived from this phenomenon:

$$
\begin{gathered}
\frac{\partial^{2} \Psi}{\partial x^{2}}+\frac{\partial^{2} \Psi}{\partial y^{2}}=k \partial_{y} T \\
\lambda\left(\frac{\partial^{2} T}{\partial x^{2}}+\frac{\partial^{2} T}{\partial y^{2}}\right)=\partial_{x} T \partial_{y} \Psi-\partial_{y} T \partial_{x} \Psi
\end{gathered}
$$

with mixed boundary conditions

$$
\begin{aligned}
\partial_{x} \Psi(x, 0) & =-\omega x^{(m-1) / 2}, \\
T(x, 0) & =T_{\omega}(x)=T_{\infty}+A x^{m}, \\
\partial_{y} \Psi(x, \infty) & =0, T(x, \infty)=T_{\infty},
\end{aligned}
$$

where $(x, y)$ is the rectangular Cartesian coordinates system. The constants $k$ and $\lambda$ depend on the density, the viscosity, and the thermal expansion coefficient of the fluid. They also depend on the permeability and the thermal diffusivity of the saturated porous medium.
In the framework of boundary layer approximations, by introducing similarity variables, we can transform the system of partial differential equations into a system of ordinary differential equations of the third order with appropriate boundary values (see [1], p.3). These two-point boundary value problems can be studied by using a shooting method (see for example $[2,3]$ ). For the auxiliary dynamical system, we refer the reader to [4]. For an integral equation, we refer the reader to [5]. For nonstandard analysis techniques, we refer the reader to [6]. For numerical method, we refer the reader to [7]. The second natural way of dealing with this problem, which is the framework of this paper, is straightly related to the coupled partial differential equations (see $[1,8,9])$.

The aim of this paper is to generalize the existence of strong solutions of the problem introduced in $[1,8,9]$. More precisely, we will give new results about the existence of strong solutions, in particular, without assuming that $\|\Psi\|_{H^{2}}$ is small (as in [8], Theorem 4). We choose the data (the function $K$ ) in the larger space inspired by other works, and we will give conditions which make each solution in $\left(C^{\infty}(\bar{\Omega})\right)^{2}$.

Let us introduce now the problem in which we are interested. Let $\Omega$ be a bounded domain of $\mathbb{R}^{2}$ whose boundary 
$\Gamma=\partial \Omega$ is sufficiently smooth and divided into two parts $\Gamma_{1}$ and $\Gamma_{2}$ such that meas $\left(\Gamma_{1}\right) \neq 0$ and

$$
\begin{aligned}
& \bar{\Gamma}_{1} \cup \bar{\Gamma}_{2}=\Gamma, \\
& \Gamma_{1} \cap \Gamma_{2}=\varnothing .
\end{aligned}
$$
by

On $\Omega$, we consider the boundary value problem defined

$$
\begin{gathered}
\Delta \Psi=K \cdot \nabla T \quad \text { a.e. } \Omega, \\
\lambda \Delta T=\nabla T \cdot \nabla \Psi^{\perp}, \quad \text { a.e. } \Omega,
\end{gathered}
$$

with boundary conditions

$$
\begin{gathered}
\Psi=0 \quad \text { on } \Gamma_{1}, \\
\frac{\partial \Psi}{\partial \nu}=0 \quad \text { on } \Gamma_{2}, \\
T=T_{\omega} \text { on } \partial \Omega,
\end{gathered}
$$

where $\lambda$ is a strictly positive constant, $v$ is the unit outward normal vector on $\partial \Omega$, and $\nabla \Psi^{\perp}=\left(\partial_{y} \Psi,-\partial_{x} \Psi\right)$.

\section{Preliminaries}

In this section, we will introduce the notations, definitions, and preliminary facts which are used throughout the paper.

2.1. Sobolev Spaces and Notations. For a positive integer $k$ and for $1 \leq p \leq \infty$, the Sobolev space is defined as follows:

$$
\begin{aligned}
W^{k, p}(\Omega) & =\left\{u \in L^{p}(\Omega), \quad \forall \alpha \in \mathbb{N}^{2} \text { with }|\alpha|\right. \\
& \left.\leq k: D^{\alpha}(u) \in L^{p}(\Omega)\right\},
\end{aligned}
$$

where $D^{\alpha}(u)$ is the $\alpha$-derivative of $u$ in the sense of distributions. For $p=2$, we denote by $H^{k}(\Omega)$ the space $W^{k, 2}(\Omega)$. The boundary space is defined as

$$
B^{k-(1 / p), p}(\partial \Omega)=\left\{\text { the space of boundary values } \varphi \text { of functions } u \in W^{k, p}(\Omega)\right\}
$$

$1<p<\infty$, equipped with the norm

$$
\|\varphi\|_{B^{k-1 / p), p}}=\inf \left\{\|u\|_{W^{k, p}}:\left.u\right|_{\partial \Omega}=\varphi\right\} .
$$

Throughout the paper, we use the following notations:

(i) $W^{k, 2}(\Omega)=H^{k}(\Omega), \quad B^{k-(1 / 2), 2}(\partial \Omega)=H^{k-(1 / 2)}(\partial \Omega)$, and $C$ is Poincaré's constant of $\Omega$

(ii) $D\left(\Omega, \Gamma_{i}\right)=\left\{u \in D(\bar{\Omega}): \supset p(u) \cap \Gamma_{i}=\varnothing\right\} \cup p(u)$

(iii) $W_{0}^{1, p}\left(\Omega, \Gamma_{i}\right)=\overline{D\left(\Omega, \Gamma_{i}\right)}{ }^{1, p}(\Omega), W_{0}^{1, p}(\Omega)=$ $W_{0}^{1, p}\left(\Omega, \Gamma_{0}\right)$, and $W_{0}^{1,2}(\Omega)=H_{0}^{1}(\Omega)$

(iv) $\|\cdot\|_{p}, 1 \leq p \leq \infty$, is the norm of $L^{p}(\Omega)$ (or the norm of $\left.L^{p}(\Omega) \times L^{p}(\Omega)\right)$

(v) $\|\cdot\|$ is the norm of $L(E, F)$, i.e., the space of linear operators from $E$ into $F$ (normed spaces)

\subsection{Variational Formulation. Let us assume}

$$
T_{\omega} \in H^{3 / 2}(\partial \Omega)
$$

Then, from the existence and uniqueness theorem for the Dirichlet problem for strong solutions (see [10], Theorem 5.13), there exists a unique function $\Theta \in H^{2}(\Omega)$ satisfying

$$
\begin{cases}\Delta \Theta=0 & \text { a.e. } \Omega \\ \Theta=T_{\omega} & \text { on } \partial \Omega\end{cases}
$$

Furthermore, $\Theta \in L^{\infty}(\Omega)$ and $\nabla \Theta \in\left(L^{4}(\Omega)\right)^{2}$. For the coefficient $K=\left(k_{1}, k_{2}\right)$, it is supposed that

$$
K \in W_{2}^{4}(\operatorname{div}, \Omega) \text {, }
$$

where

$$
W_{q}^{p}(\operatorname{div}, \Omega)=\left\{f \in L^{p}\left(\Omega, \mathbb{R}^{2}\right): \operatorname{div}(K) \in L^{q}(\Omega)\right\} .
$$

Let us notice that $D\left(\bar{\Omega}, \mathbb{R}^{2}\right)$ is dense in $W_{2}^{4}(\operatorname{div}, \Omega$ ) (see [11], Proposition 3.57). Let us set $H=T-\Theta$. Then, $(\Psi, T)$ is the solution of problem (5)-(7) if and only if $(\Psi, H)$ is the solution of the problem

$$
\begin{aligned}
\Delta \Psi & =K \cdot \nabla(H+\Theta) \quad \text { a.e. } \Omega, \\
\lambda \Delta H & =\nabla H \cdot \nabla \Psi^{\perp}+\nabla \Theta \cdot \nabla \Psi^{\perp} \quad \text { a.e. } \Omega,
\end{aligned}
$$

with boundary conditions

$$
\begin{gathered}
\Psi=0 \quad \text { on } \Gamma_{1}, \\
\frac{\partial \Psi}{\partial \nu}=0 \quad \text { on } \Gamma_{2}, \\
H=0 \quad \text { on } \partial \Omega .
\end{gathered}
$$

Let us assume that $(\Psi, H) \in\left(H^{2}(\Omega)\right)^{2}$ is a solution of problem (15)-(18). Multiplying equation (15) by a function $u \in H_{0}^{1}\left(\Omega, \Gamma_{1}\right)$ and equation (16) by a function $v \in H_{0}^{1}(\Omega)$, integrating on $\Omega$, and using Green's formula, we yield

$\left\{\begin{array}{l}(\nabla \Psi, \nabla u)=-(K \cdot \nabla(H+\Theta), u), \quad \forall u \in H_{0}^{1}\left(\Omega, \Gamma_{1}\right), \\ \lambda(\nabla H, \nabla v)=-\left(v \nabla H, \nabla \Psi^{\perp}\right)-\left(v \nabla \Theta, \nabla \Psi^{\perp}\right), \quad \forall v \in H_{0}^{1}(\Omega),\end{array}\right.$

where 


$$
(f, g)=\int_{\Omega} f g
$$

\section{Main Results}

We start with the following lemma which plays a key role in our main results.

Lemma 1. Let $\Psi \in W^{1,4}(\Omega)$. The function $H \equiv 0$ is the unique solution of problem

$$
\left\{\begin{array}{l}
\lambda \Delta H-\nabla H \cdot \nabla \Psi^{\perp}=0 \quad \text { a.e } \Omega, \\
H=0 \text { on } \partial \Omega,
\end{array}\right.
$$

in the space $H^{2}(\Omega) \cap H_{0}^{1}(\Omega)$.

Proof. It is clear that $H \equiv 0$ is the solution of problem (21). Then, if $H \in H^{2}(\Omega) \cap H_{0}^{1}(\Omega)$ satisfies $\lambda \Delta H-\nabla H \cdot \nabla \Psi^{\perp}=0$, multiplying the members of this equation by $H$, we get

$$
\lambda H \Delta H=H \nabla H \cdot \nabla \Psi^{\perp}, \quad \text { a.e. on } \Omega \text {. }
$$

By integration on $\Omega$, we get

$$
\begin{aligned}
\lambda \int_{\Omega} H \Delta H & =-\lambda\|\nabla H\|_{2}^{2} \\
& =\int_{\Omega} H \nabla H \cdot \nabla \Psi^{\perp} \\
& =\left(\nabla H \cdot \nabla \Psi^{\perp}, H\right) .
\end{aligned}
$$

On the other hand, we have

$$
\begin{aligned}
\nabla H \cdot \nabla \Psi^{\perp} & =\operatorname{div}\left(H \nabla \Psi^{\perp}\right)-H \operatorname{div}\left(\nabla \Psi^{\perp}\right) \\
& =\operatorname{div}\left(H \nabla \Psi^{\perp}\right),
\end{aligned}
$$

because $\operatorname{div}\left(\nabla \Psi^{\perp}\right)=0$, in the sense of distributions. Since $\nabla H \cdot \nabla \Psi^{\perp} \in L^{2}(\Omega), \operatorname{div}\left(H \nabla \Psi^{\perp}\right) \in L^{2}(\Omega)$, and hence, by the density of $D(\Omega)$ in $H_{0}^{1}(\Omega)$ (when $\Omega$ is at least a Lipschitz open set), we have

$$
\begin{aligned}
-\lambda\|\nabla H\|_{2}^{2} & =\left(\operatorname{div}\left(H \cdot \nabla \Psi^{\perp}\right), H\right)_{H^{-1}(\Omega), H_{0}^{1}(\Omega)} \\
& =-\left(H \cdot \nabla \Psi^{\perp}, \nabla H\right) \\
& =-\left(\nabla H \cdot \nabla \Psi^{\perp}, H\right) \\
& =\lambda\|\nabla H\|_{2}^{2},
\end{aligned}
$$

that is, $\|\nabla H\|_{2}=0$. Thus, $H=0$.

Remark 1. From the proof of the previous lemma, we observe that for all $H \in H^{2}(\Omega) \cap H_{0}^{1}(\Omega)$ and $\Psi \in W^{1,4}(\Omega)$, we have

$$
\left(H, \nabla H \cdot \nabla \Psi^{\perp}\right)=0 .
$$

Thus, by the density of $D(\Omega)$ in $H_{0}^{1}(\Omega)$ and by the embedding $H^{1}(\Omega) \rightarrow L^{4}(\Omega)$, we can easily show that for all $H \in H_{0}^{1}(\Omega)$ and $\Psi \in W^{1,4}(\Omega)$, we have

$$
\left(H, \nabla H \cdot \nabla \Psi^{\perp}\right)=0 .
$$

Now, we will prove the following proposition which plays an important role in proving our main results.

Proposition 1. Let us assume that $\partial \Omega$ is of class $C^{1,1}$ and $\Psi \in W^{1,4}(\Omega)$. Then, for all $f \in L^{2}(\Omega)$, the equation

$$
\lambda \Delta H-\nabla H \cdot \nabla \Psi^{\perp}=f \quad \text { a.e. } \Omega
$$

has exactly one solution $H$ in the space $H^{2}(\Omega) \cap H_{0}^{1}(\Omega)$.

Proof. Let us consider

$$
T_{\Psi}: H^{2}(\Omega) \cap H_{0}^{1}(\Omega) \longrightarrow L^{2}(\Omega),
$$

defined by

$$
\begin{gathered}
T_{\Psi}(H)=\nabla H \cdot \nabla \Psi^{\perp}, \\
T: H^{2}(\Omega) \cap H_{0}^{1}(\Omega) \longrightarrow L^{2}(\Omega),
\end{gathered}
$$

where the operator is defined by

$$
T(H)=\lambda \Delta H
$$

Then, it is clear that for all $H \in H^{2}(\Omega) \cap H_{0}^{1}(\Omega)$, we have

$$
\lambda \Delta H-\nabla H \cdot \nabla \Psi^{\perp}=f \Longleftrightarrow T(H)-T_{\Psi}(H)=f .
$$

From the existence and uniqueness theorems for the Dirichlet problem for strong solutions (see [12], Theorem 9.15, Lemma 9.17, problem 9.8) combined with the open mapping theorem (see [13], Corollary 2.7), the operator $T$ is invertible. On the other hand, we can write $T_{\Psi}=\varphi_{2} \circ \varphi_{1}$ where

$$
\varphi_{1}: H^{2}(\Omega) \cap H_{0}^{1}(\Omega) \longrightarrow\left(L^{4}(\Omega)\right)^{2},
$$

defined by

$$
\varphi_{1}(H)=\left(\bar{i}_{c} \circ j\right)(H)
$$

with $j$ as the operator from $H^{2}(\Omega) \cap H_{0}^{1}(\Omega)$ into $\left(H^{1}(\Omega)\right)^{2}$ defined by $j(H)=\nabla H$ and $\bar{i}_{c}=\left(i_{c}, i_{c}\right)$, where $i_{c}: H^{1}(\Omega) \rightarrow L^{4}(\Omega)$, and

$$
\varphi_{2}:\left(L^{4}(\Omega)\right)^{2} \longrightarrow L^{2}(\Omega)
$$

defined by

$$
\varphi_{2}(\xi)=\xi \cdot \nabla \Psi^{\perp}
$$

Then, $T_{\Psi}$ is compact. Indeed, $\varphi_{1}$ is continuous because $i_{c}$ and $j$ are continuous, and the operator $i_{c}$ is compact (Rellich-Kondrachov theorem); it is clear that $\varphi_{2}$ is continuous. Now, it is easy to show that for all $H \in H^{2}(\Omega) \cap H_{0}^{1}(\Omega)$, (32) holds, if and only if

$$
H-\left(T^{-1} \circ T_{\Psi}\right)(H)=T^{-1}(f)
$$

holds. By Lemma (21), $H \equiv 0$ is the unique solution of equation

$$
T(H)-T_{\Psi}(H)=0,
$$


in the space $H^{2}(\Omega) \cap H_{0}^{1}(\Omega)$. Hence, from the Fredholm alternative, equation (28) has exactly one solution $H \in H^{2}(\Omega) \cap H_{0}^{1}(\Omega)$. Besides

$$
\|H\|_{H^{2}} \leq\left\|\left(I-T^{-1} \circ T_{\Psi}\right)^{-1}\right\| \cdot\left\|T^{-1}\right\| \cdot\|f\|_{2},
$$

as needed.

Lemma 2. Under the assumptions of the previous proposition, the mapping $L$ from $W^{1,4}(\Omega)$ into $H_{0}^{1}(\Omega)$ is defined by $L(\Psi)=H$ where

$$
\left\{\begin{array}{l}
\lambda \Delta H-\nabla H \cdot \nabla \Psi^{\perp}=\nabla \Theta \cdot \nabla \Psi^{\perp} \quad \text { a.e } \Omega \\
H=0 \quad \text { on } \partial \Omega
\end{array}\right.
$$

(Then, $L=i \circ\left(I-T^{-1} \circ T_{\Psi}\right)^{-1}$ with $\left.i: H^{2}(\Omega) \rightarrow H^{1}(\Omega)\right) \quad$ is continuous. Moreover, there exists $R>0$ such that for all $\Psi \in W^{1,4}(\Omega)$, if $L(\Psi)=H$,

$$
|H+\Theta| \leq R
$$

Proof. Let $\Psi \in W^{1,4}(\Omega)$ and let $H \in H^{2}(\Omega) \cap H_{0}^{1}(\Omega)$ be the unique solution of problem (40). Let $\left(\Psi_{n}\right)_{n}$ be a sequence of elements in $D(\bar{\Omega})$ converging to $\Psi$ in $W^{1,4}(\Omega)$. Then, there exists a sequence $\left(H_{n}\right)_{n}$ of elements in $H^{2}(\Omega) \cap H_{0}^{1}(\Omega)$ satisfying

$$
\left\{\begin{array}{l}
\lambda \Delta H_{n}-\nabla H_{n} \cdot \nabla \Psi_{n}^{\perp}=\nabla \Theta \cdot \nabla \Psi_{n}^{\perp} \\
H_{n}=0 \text { on } \partial \Omega
\end{array}\right.
$$

Then, for all $n \in \mathbb{N}, H_{n}+\Theta$ is the solution of the problem

$$
\left\{\begin{array}{l}
\lambda \Delta W-\nabla W \cdot \nabla \Psi_{n}^{\perp}=0 \\
W=T_{\omega} \text { on } \partial \Omega
\end{array}\right.
$$

By the weak maximum principle theorem (see [12], Theorem 8.1), for all $n \in \mathbb{N}$, we have

$$
-\sup _{\partial \Omega}\left|T_{\omega}\right| \leq H_{n}+\Theta \leq \sup _{\partial \Omega}\left|T_{\omega}\right|,
$$

and by variational formulation (system (19)), we have

$\begin{cases}\lambda(\nabla H, \nabla v)=-\left(v \nabla H, \nabla \Psi^{\perp}\right)-\left(v \nabla \Theta, \nabla \Psi^{\perp}\right), & \forall v \in H_{0}^{1}(\Omega), \\ \lambda\left(\nabla H_{n}, \nabla v\right)=-\left(v \nabla H_{n}, \nabla \Psi_{n}^{\perp}\right)-\left(v \nabla \Theta, \nabla \Psi_{n}^{\perp}\right), & \forall v \in H_{0}^{1}(\Omega) .\end{cases}$

Replacing $v$ by $H-H_{n}$ and using Remark 1, we give

$$
\begin{aligned}
\lambda\left\|\nabla\left(H_{n}-H\right)\right\|_{2}^{2}= & -\left(\left(H-H_{n}\right) \nabla H, \nabla \Psi^{\perp}\right) \\
& +\left(\left(H-H_{n}\right) \nabla H_{n}, \nabla \Psi_{n}^{\perp}\right) \\
& +\left(H-H_{n} \cdot \nabla \Theta, \nabla\left(\Psi_{n}-\Psi\right)^{\perp}\right) \\
= & \left(H_{n} \nabla H, \nabla \Psi^{\perp}\right)+\left(H \nabla H_{n}, \nabla \Psi_{n}^{\perp}\right) \\
& +\left(H-H_{n} \cdot \nabla \Theta, \nabla\left(\Psi_{n}-\Psi\right)^{\perp}\right) .
\end{aligned}
$$

On the other hand, for $W=H-H_{n}$, we have

$$
\begin{aligned}
\left(W \cdot \nabla \Theta, \nabla \Psi^{\perp}\right)= & \int_{\Omega}\left(W \operatorname{div}\left(\Theta \cdot \nabla \Psi^{\perp}\right)\right. \\
& \left.-W \cdot\left(\Theta \cdot \operatorname{div}\left(\nabla \Psi^{\perp}\right)\right)\right) \\
= & -\int_{\Omega} \Theta \cdot \nabla W \cdot \nabla \Psi^{\perp}, \\
= & -\left(\Theta \nabla W, \nabla \Psi^{\perp}\right) .
\end{aligned}
$$

Similarly, $\left(H \nabla H_{n}, \nabla \Psi_{n}^{\perp}\right)=-\left(H_{n} \nabla H, \nabla \Psi_{n}^{\perp}\right)$. We replace the previous inequality in inequality (46), and we use Remark 1, to get

$$
\begin{aligned}
\lambda\left\|\nabla\left(H-H_{n}\right)\right\|_{2}^{2}= & \left(H_{n} \nabla H, \nabla \Psi^{\perp}\right)-\left(H_{n} \nabla H, \nabla \Psi_{n}^{\perp}\right) \\
& +\left(\Theta \nabla\left(H-H_{n}\right), \nabla\left(\Psi-\Psi_{n}\right)^{\perp}\right), \\
= & \left(H_{n} \nabla H, \nabla\left(\Psi-\Psi_{n}\right)^{\perp}\right) \\
& +\left(\Theta \nabla\left(H-H_{n}\right), \nabla\left(\Psi-\Psi_{n}\right)^{\perp}\right) \\
= & \left(H_{n} \nabla\left(H-H_{n}\right), \nabla\left(\Psi-\Psi_{n}\right)^{\perp}\right) \\
& +\left(\Theta \nabla\left(H-H_{n}\right), \nabla\left(\Psi-\Psi_{n}\right)^{\perp}\right) \\
= & \left(\left(H_{n}+\Theta\right) \nabla\left(H-H_{n}\right), \nabla\left(\Psi-\Psi_{n}\right)^{\perp}\right), \\
\leq & \left\|H_{n}+\Theta\right\|_{\infty}\left\|\nabla\left(H_{n}-H\right)\right\|_{2}\left\|\nabla\left(\Psi_{n}-\Psi\right)\right\|_{2} .
\end{aligned}
$$

Thus,

$\left\|\nabla\left(H-H_{n}\right)\right\|_{2} \leq \frac{R}{\lambda}\left\|\nabla\left(\Psi_{n}-\Psi\right)\right\|_{2} \longrightarrow 0 \quad$ as $n \longrightarrow \infty$,

with

$$
R=\sup _{\partial \Omega}\left|T_{\omega}\right| \cdot
$$

Then, there exists a subsequence $\left(H_{n_{k}}\right)_{k}$ of the sequence $\left(H_{n}\right)_{n}$ converging almost everywhere to $H$. That is,

$$
|H+\Theta| \leq R .
$$

From inequality (49), we get, for all $\Psi_{1}, \Psi_{2} \in W^{1,4}(\Omega)$,

$$
\begin{aligned}
\left\|\nabla\left(H_{1}-H_{2}\right)\right\|_{2} & \leq \frac{R}{\lambda}\left\|\nabla\left(\Psi_{1}-\Psi_{2}\right)\right\|_{2} \\
& \leq C^{\prime \prime}\left\|\nabla\left(\Psi_{1}-\Psi_{2}\right)\right\|_{4},
\end{aligned}
$$

where $C^{\prime \prime}>0$ depends only on $\Omega, R$, and $\lambda$, as needed.

\section{Remarks 2}

(1) The main advantage of Proposition 1 (particularly, Fredholm's alternative) is the generalization of the results ([8], Theorem 1 and Theorem 4, and [1], Theorem 4), and more precisely, it leads us to show that there exist strong solutions to problem (15)-(18) without assuming that $\|\Psi\|_{H^{2}}$ is bounded by certain constants and without assuming that $\|K \cdot \nabla \Theta\|_{2}$ is quite small. 
(2) The previous lemma is similar to Lemma 3.1 in [8], but in this paper, we have seen a new technique for its proof (it is proved by a weak maximum principle).

The following proposition is useful.

Proposition 2. Let us assume, in addition to assumptions (11)-(13), that $\partial \Omega$ is of class $C^{1,1}, \Gamma_{1}=\partial \Omega$, and $K \in(D(\bar{\Omega}))^{2}$. Then, problem (15)-(18) have at least one solution $(\Psi, H)$ in the space $\left(W^{2,4}(\Omega)\right)^{2}$.

Proof. For all $H \in H_{0}^{1}(\Omega)$, we have $K \cdot \nabla(H+\Theta) \in L^{2}(\Omega)$. Then, from the existence and uniqueness theorem for the Dirichlet problem for strong solutions ([12], Theorem 9.15), the problem

$$
\left\{\begin{array}{l}
\Delta \Psi=K \cdot \nabla(H+\Theta) \text { a.e. } \Omega \\
\Psi=0 \text { on } \partial \Omega
\end{array}\right.
$$

has exactly one solution $\Psi \in H^{2}(\Omega)$. Besides, there exists $\bar{C}>0$ such that

$$
\|\Psi\|_{H^{2}} \leq \bar{C}\|K \cdot \nabla(H+\Theta)\|_{2}
$$

(see [12], Lemma 9.17). Let us denote by $\Lambda$ the application from $H_{0}^{1}(\Omega)$ into $H^{2}(\Omega)$ defined by $\Lambda(H)=\Psi$, and let us consider the application $J: H_{0}^{1}(\Omega) \longrightarrow H_{0}^{1}(\Omega)$ defined by

$$
J=L \circ i \circ \Lambda \text {, }
$$

with $L$ given in Lemma 2 and $i: H^{2}(\Omega) \rightarrow W^{1,4}(\Omega)$. Then, we have

$$
J(H)=L \circ i \circ \Lambda(H), \quad \forall H \in H_{0}^{1}(\Omega),
$$

that is, the set of the solutions of problem (15)-(18) is $\left\{(\Lambda(H), H)\right.$ such that $H \in H^{2}(\Omega) \cap H_{0}^{1}(\Omega)$ and $\left.J(H)=H\right\}$.

Then, $J$ is continuous and compact. Indeed, $L$ and $\Lambda$ are continuous because for $\Psi_{i}=\Lambda\left(H_{i}\right), i=1,2$, we have

$$
\left\|\Lambda\left(\Psi_{1}\right)-\Lambda\left(\Psi_{2}\right)\right\|_{H^{2}} \leq \bar{C}\|K\|_{\infty}\left\|\nabla\left(H_{1}-H_{2}\right)\right\|_{2},
$$

and the continuity of $L$ has been proved in Lemma 2. By the Rellich-Kondrachov theorem, we can easily show that $i$ is compact. Now, let $H$ be in the set

$$
A=\left\{H \in H_{0}^{1}(\Omega): H=\varepsilon J(H) \quad \text { for some } \varepsilon \in[0,1]\right\} .
$$

Then, there exists $\varepsilon \in[0,1]$ such that

$$
\left\{\begin{array}{l}
\Delta \Psi=\varepsilon K \cdot \nabla H+K \cdot \nabla \Theta, \quad \text { a.e } \Omega, \\
\lambda \Delta H-\nabla H \cdot \nabla \Psi^{\perp}=\nabla \Theta \cdot \nabla \Psi^{\perp}, \quad \text { a.e } \Omega, \\
\Psi=0 \text { on } \partial \Omega, \\
H=0 \text { on } \partial \Omega .
\end{array}\right.
$$

By the variational formulation, the previous problem is equivalent to $\begin{cases}(\nabla \Psi, \nabla u)=-(K \cdot \nabla(\varepsilon H+\Theta), u), & \forall u \in H_{0}^{1}(\Omega), \\ \lambda(\nabla H, \nabla v)=-\left(v \nabla H, \nabla \Psi^{\perp}\right)-\left(v \nabla \Theta, \nabla \Psi^{\perp}\right), & \forall v \in H_{0}^{1}(\Omega) .\end{cases}$

From the first equation in system (61), we have

$$
\begin{aligned}
\|\nabla \Psi\|_{2}^{2} & =-\varepsilon(K \cdot \nabla H, \Psi)-(K \cdot \nabla \Theta, \Psi) \\
& =\varepsilon((H \operatorname{div}(K), \Psi)-(\operatorname{div}(H K), \Psi))-(K \cdot \nabla \Theta, \Psi) \\
& =\varepsilon((H \operatorname{div}(K), \Psi)+(H K, \nabla \Psi))-(K \cdot \nabla \Theta, \Psi) \\
& \leq R^{\prime}\|\operatorname{div}(K)\|_{2}\|\Psi\|_{2}+R^{\prime}\|K\|_{2}\|\nabla \Psi\|_{2}+\|K\|_{4}\|\nabla \Theta\|_{4}\|\Psi\|_{2},
\end{aligned}
$$

with $R^{\prime}=R^{\prime}(\Theta, \Omega)$. Hence,

$$
\|\nabla \Psi\|_{2} \leq C R^{\prime}\|\operatorname{div}(K)\|_{2}+R^{\prime}\|K\|_{2}+C\|K\|_{4}\|\nabla \Theta\|_{4} .
$$

Now, from the second equation in system (61), since $\left(H \nabla H, \nabla \Psi^{\perp}\right)=0 \quad$ (see Remark 1) and $\left(H \nabla \Theta, \nabla \Psi^{\perp}\right)=-\left(\Theta \nabla H, \nabla \Psi^{\perp}\right)$ (the same method as the one used to get inequality (47)), we have

$$
\|\nabla H\|_{2} \leq \frac{\|\Theta\|_{\infty}}{\lambda}\|\nabla \Psi\|_{2} .
$$

From inequalities (63) and (64), we deduce that $\|H\|_{H^{1}}<\infty$, i.e., $A$ is bounded. From Schäfer's fixed-point theorem (see [14], Theorem 11.1, p.59), we deduce that $J$ has at least one fixed point $H \in H^{2}(\Omega) \cap H_{0}^{1}(\Omega)$. That is, $(\Lambda(H), H)$ is the strong solution of problem (15)-(18). Furthermore, $K \cdot \nabla(H+\Theta) \in L^{4}(\Omega)$. Thus, $\Psi \in W^{2,4}(\Omega)$, i.e., $\nabla \Psi \in\left(L^{\infty}(\Omega)\right)^{2}$. Hence, $H \in W^{2,4}(\Omega)$, as needed.

Remark 3. From the previous proof, it is observed that under the assumptions

$$
\begin{aligned}
K & \in L^{\infty}(\Omega) \times L^{\infty}(\Omega), \\
\operatorname{div}(K) & \in L^{\infty}(\Omega),
\end{aligned}
$$

the previous result (the previous proposition) is still valid, which is the generalization of ([8], Theorem 1 and Theorem 4). In addition, Proposition 1 helps us to generalize the previous result in the case where $K \in W_{2}^{4}(\operatorname{div}, \Omega)$.

Now, we are going to generalize the previous result in the case where $K \in W_{2}^{4}(\operatorname{div}, \Omega)$. To this end, we start with the following remark.

Remark 4. Let $(\Psi, H) \in\left(W^{2,4}(\Omega) \cap W_{0}^{1,4}(\Omega)\right)^{2}$ be a solution of the problem

$$
\left\{\begin{array}{l}
\Delta \Psi=K \cdot \nabla H+K \cdot \nabla \Theta, \quad \text { a.e. } \Omega, \\
\lambda \Delta H-\nabla H \cdot \nabla \Psi^{\perp}=\nabla \Theta \cdot \nabla \Psi^{\perp}, \quad \text { a.e. } \Omega, \\
\Psi=0 \quad \text { on } \partial \Omega \\
H=0 \quad \text { on } \partial \Omega .
\end{array}\right.
$$

Then, from the first equation with boundary conditions, for all $u \in H_{0}^{1}(\Omega)$, we have 


$$
\begin{aligned}
\int_{\Omega} \nabla \Psi \cdot \nabla u= & -(K \cdot \nabla(H+\Theta), u) \\
& =-\int_{\Omega} K \cdot \nabla H \cdot u-\int_{\Omega} K \cdot \nabla \Theta \cdot u \\
& =\int_{\Omega}(H \operatorname{div}(K)-\operatorname{div}(H K)) \cdot u-\int_{\Omega} K \cdot \nabla \Theta \cdot u \\
& =\int_{\Omega} H \operatorname{div}(K) \cdot u-\int_{\Omega} \operatorname{div}(H K) \cdot u-\int_{\Omega} K \cdot \nabla \Theta \cdot u \\
& \int_{\Omega} H \operatorname{div}(K) \cdot u+\int_{\Omega} H K \cdot \nabla u-\int_{\Omega} K \cdot \nabla \Theta \cdot u \\
= & (H \operatorname{div}(K), u)+(H K, \nabla u)-(K \cdot \nabla \Theta, u) .
\end{aligned}
$$

Now, from the second equation in system (66) with boundary conditions, for all $v \in H_{0}^{1}(\Omega)$, we have

$$
\lambda(\nabla H, \nabla v)=-\left(v \nabla H, \nabla \Psi^{\perp}\right)-\left(v \nabla \Theta, \nabla \Psi^{\perp}\right) .
$$

On the other hand, we have

$$
\left(H \nabla v, \nabla \Psi^{\perp}\right)=-\left(v \nabla H, \nabla \Psi^{\perp}\right),
$$

the same method as the one used to get inequality (47). Then, we have

$$
\lambda(\nabla H, \nabla v)=\left(H \nabla v, \nabla \Psi^{\perp}\right)-\left(v \nabla \Theta, \nabla \Psi^{\perp}\right) .
$$

Thereby, if $(\Psi, H) \in\left(W^{2,4}(\Omega) \cap W_{0}^{1,4}(\Omega)\right)^{2}$ is a solution of problem (66), then for all $u, v \in H_{0}^{1}(\Omega)$, we have

$$
\begin{aligned}
(\nabla \Psi, \nabla u) & =(K \cdot \nabla(H+\Theta), u) \\
& =(H \operatorname{div}(K), u)+(H K, \nabla u)-(K \cdot \nabla \Theta, u), \\
\lambda(\nabla H, \nabla v) & =\left(H \nabla v, \nabla \Psi^{\perp}\right)-\left(v \nabla \Theta, \nabla \Psi^{\perp}\right) .
\end{aligned}
$$

Now, we will prove the following result.

Theorem 1. Let us assume, in addition to assumptions (11)-(13), that $\partial \Omega$ is of class $C^{1,1}$ and $\Gamma_{1}=\partial \Omega$. Then, problem (15)-(18) have at least one solution $(\Psi, H)$ in the space $\left(W^{2,4}(\Omega) \cap W_{0}^{1,4}(\Omega)\right)^{2}$.

Proof. Let $\left(K_{n}\right)_{n} \subseteq D(\bar{\Omega})$ be a sequence converging to $K$ in $W_{2}^{4}(\operatorname{div}, \Omega)$. Then, from Proposition 2 , there exists $\left(\Psi_{n}, H_{n}\right)_{n} \subseteq\left(W^{2,4}(\Omega) \cap W_{0}^{1,4}(\Omega)\right)^{2}$ which is bounded in $\left(H^{1}(\Omega)\right)^{2}$ and satisfying

$$
\left\{\begin{array}{l}
\Delta \Psi_{n}=K_{n} \cdot \nabla H_{n}+K_{n} \cdot \nabla \Theta, \quad \text { a.e } \Omega, \\
\lambda \Delta H_{n}-\nabla H_{n} \cdot \nabla \Psi_{n}^{\perp}=\nabla \Theta \cdot \nabla \Psi_{n}^{\perp}, \quad \text { a.e } \Omega, \\
\Psi_{n}=0 \quad \text { on } \partial \Omega \\
H_{n}=0 \quad \text { on } \partial \Omega .
\end{array}\right.
$$

By the compactness of the embedding $H^{1}(\Omega) \rightarrow L^{p}(\Omega), p \geq 2$, there exists a subsequence $\left(\Psi_{n_{k}}, H_{n_{k}}\right)_{k}$ of the sequence $\left(\Psi_{n}, H_{n}\right)_{n}$ converging weakly in $\left(H^{1^{k}}(\Omega)\right)^{2}$ and strongly in $L^{p}(\Omega), p \geq 2$, to an element denoted $\left(\Psi_{*}, H_{*}\right)$, and we have $H_{*} \in L^{\infty}(\Omega)$. Now, we shall complete the proof in three steps.
Step 1. $\left(\Psi_{n_{k}}, H_{n_{k}}\right)_{k}$ converges in $H^{1}$ to $\left(\Psi_{*}, H_{*}\right)$. Indeed, from Remark 4, for all $u \in D(\Omega)$, we have

$$
\begin{aligned}
\left(\nabla\left(\Psi_{n_{k+p}}-\Psi_{n_{k}}\right), \nabla u\right)= & \left(H_{n_{k+p}} \operatorname{div}\left(K_{n_{k+p}}\right)-H_{n_{k}} \operatorname{div}\left(K_{n_{k}}\right), u\right) \\
& +\left(H_{n_{k+p}} K_{n_{k+p}}-H_{n_{k}} K_{n_{k}}, u\right) \\
& -\left(\left(K_{n_{k+p}}-K_{n_{k}}\right) \nabla \Theta, u\right) \\
= & \left(H_{n_{k+p}} \operatorname{div}\left(K_{n_{k+p}}-K_{n_{k}}\right), u\right) \\
& +\left(\operatorname{div}\left(K_{n_{k}}\right)\left(H_{n_{k+p}}-H_{n_{k}}\right), u\right) \\
& +\left(H_{n_{k+p}}\left(K_{n_{k+p}}-K_{n_{k}}\right), \nabla u\right) \\
& +\left(K_{n_{k}}\left(H_{n_{k+p}}-H_{n_{k}}\right), \nabla u\right) \\
& -\left(\left(K_{n_{k+p}}-K_{n_{k}}\right) \nabla \Theta, u\right) .
\end{aligned}
$$

Then, for $u=\Psi_{n_{k+p}}-\Psi_{n_{k}}$, we have

$$
\begin{aligned}
\left\|\nabla\left(\Psi_{n_{k+p}}-\Psi_{n_{k}}\right)\right\|_{2}^{2} \leq & \left\|H_{n_{k+p}}\right\|_{\infty}\left\|\operatorname{div}\left(K_{n_{k+p}}-K_{n_{k}}\right)\right\|\left\|_{2}\right\| \Psi_{n_{k+p}}-\Psi_{n_{k}} \|_{2} \\
& +\left\|\operatorname{div}\left(K_{k}\right)\right\|_{2}\left\|H_{n_{k+p}}-H_{n_{k}}\right\|_{4}\left\|\Psi_{n_{k+p}}-\Psi_{k}\right\|_{4} \\
& +\left\|H_{n_{k+p}}\right\|_{\infty}\left\|K_{n_{k+p}}-K_{n_{k}}\right\|_{2}\left\|\nabla\left(\Psi_{n_{k+p}}-\Psi_{n_{k}}\right)\right\|_{2} \\
& +\left\|K_{n_{k}}\right\|\left\|_{4}\right\| H_{n_{k+p}}-H_{n_{k}}\|\| \nabla\left(\Psi_{4}\left\|\left(\Psi_{n_{k+p}}-\Psi_{n_{k}}\right)\right\|_{2}\right. \\
& +\left\|K_{n_{k+p}}-K_{n_{k}}\right\|_{4}\|\nabla \Theta\|_{4}\left\|\nabla\left(\Psi_{n_{k+p}}-\Psi_{n_{k}}\right)\right\|_{2} .
\end{aligned}
$$

By Lemma 2 and the fact that $\left(K_{n}\right)_{n}$ is convergent in $W_{2}^{4}(\operatorname{div}, \Omega)$, we get that there are strictly positive constants $C_{1}, C_{2}$, and $C_{3}$, such that

$$
\begin{aligned}
\left\|\nabla\left(\Psi_{n_{k+p}}-\Psi_{n_{k}}\right)\right\|_{2} \leq & C_{1}\left\|\operatorname{div}\left(K_{n_{k+p}}-K_{n_{k}}\right)\right\|_{2} \\
& +C_{2}\left\|K_{n_{k+p}}-K_{n_{k}}\right\|_{4}+C_{3}\left\|H_{n_{k+p}}-H_{n_{k}}\right\|_{4} .
\end{aligned}
$$

That is, $\left(\Psi_{n_{k}}\right)_{k}$ converges strongly to $\Psi_{*}$. On the other hand, for all $v \in H_{0}^{1}(\Omega)$, we have

$$
\lambda\left(\nabla H_{n_{k}}, \nabla v\right)=\left(H_{n_{k}} \nabla v, \nabla \Psi_{n_{k}}^{\perp}\right)-\left(v \nabla \Theta, \nabla \Psi_{n_{k}}^{\perp}\right),
$$

By the same process as the one used to obtain inequality (52), we have

$$
\left\|\nabla\left(H_{n_{k+p}}-H_{n_{k}}\right)\right\|_{2} \leq \frac{\|\Theta\|_{\infty}}{\lambda}\left\|\nabla\left(\Psi_{n_{k+p}}-\Psi_{n_{k}}\right)\right\|_{2},
$$

that is, $\left(H_{n_{k}}\right)_{k}$ converges strongly to $H_{*}$.

Step 2. $\left(\Psi_{*}, H_{*}\right)$ satisfies systems (71) and (72). Indeed, for all $u \in D(\Omega)$, we have 


$$
\begin{aligned}
\left(K_{n_{k}} \cdot \nabla\left(H_{n_{k}}+\Theta\right), u\right)= & \left(K_{n_{k}} \cdot \nabla\left(H_{n_{k}}-H_{*}\right), u\right) \\
& +\left(K_{n_{k}} \cdot \nabla\left(H_{*}+\Theta\right), u\right) .
\end{aligned}
$$

For all $u \in D(\Omega)$, we have

$$
\begin{gathered}
\left|\left(K_{n_{k}} \nabla\left(H_{n_{k}}-H_{*}\right), u\right)\right| \leq\left\|K_{n_{k}}\right\|\left\|_{4}\right\| \nabla\left(H_{n_{k}}-H_{*}\right)\left\|_{2}\right\| u \|_{4} \longrightarrow 0 \\
\left|\left(K_{n_{k}} \cdot \nabla\left(H_{*}+\Theta\right), u\right)-\left(K \cdot \nabla\left(H_{*}+\Theta\right), u\right)\right| \leq\left\|K_{n_{k}}-K\right\|_{4}\left\|\nabla\left(H_{*}+\Theta\right)\right\|_{2}\|u\|_{4} \longrightarrow 0 .
\end{gathered}
$$

Taking the limit as $k \longrightarrow \infty$ in $(71)$ when $(\Psi, H, K)=$ $\left(\Psi_{n_{k}}, H_{n_{k}}, K_{n_{k}}\right)$ gives

$\left(\nabla \Psi_{*}, \nabla u\right)=\left(K \cdot \nabla\left(H_{*}+\Theta\right), u\right), \quad \forall u \in D(\Omega)$.

Now, for all $v \in H_{0}^{1}(\Omega)$, we have

$$
\begin{aligned}
\left(v \nabla H_{n_{k}}, \nabla \Psi_{n_{k}}^{\perp}\right)= & -\left(H_{n_{k}} \cdot \nabla v, \nabla \Psi_{n_{k}}^{\perp}\right) \\
= & -\left(H_{n_{k}} \cdot \nabla v, \nabla\left(\Psi_{n_{k}}-\Psi_{*}\right)^{\perp}\right) \\
& -\left(H_{n_{k}} \cdot \nabla v, \nabla \Psi_{*}^{\perp}\right) \\
& \longrightarrow-\left(H_{*} \cdot \nabla v, \nabla \Psi_{*}^{\perp}\right),
\end{aligned}
$$

because

$$
\left|\left(H_{n_{k}} \cdot \nabla v, \nabla\left(\Psi_{n_{k}}-\nabla \Psi_{*}\right)^{\perp}\right)\right| \leq R^{\prime}\|\nabla v\|_{2}\left\|\nabla\left(\Psi_{n_{k}}-\Psi_{*}\right)^{\perp}\right\|_{2}
$$$$
\longrightarrow 0 \text {. }
$$

By the dominated convergence theorem, we have

$$
\left(H_{n_{k}} \cdot \nabla v, \nabla \Psi_{*}^{\perp}\right) \longrightarrow\left(H_{*} \nabla v, \nabla \Psi_{*}^{\perp}\right) .
$$

Obviously,

$$
\left(\nabla\left(H_{n_{k}}-H_{*}\right), \nabla v\right),\left(\nu \nabla \Theta, \nabla\left(\Psi_{n_{k}}-\Psi_{*}\right)^{\perp}\right) \longrightarrow 0 .
$$

Taking the limit in (77) as $k \longrightarrow \infty$ yields

$$
\lambda\left(\nabla H_{*}, \nabla v\right)=\left(H_{*} \nabla v, \nabla \Psi_{*}^{\perp}\right)-\left(v \nabla \Theta, \nabla \Psi_{*}^{\perp}\right) .
$$

Step 3. $\left(\Psi_{*}, H_{*}\right) \in\left(W^{2,4}(\Omega)\right)^{2}$. Indeed

$$
\left(\nabla \Psi_{*}, \nabla u\right)=-\left(K \cdot \nabla\left(H_{*}+\Theta\right), u\right), \quad \forall u \in D(\Omega) .
$$

Since $K \in\left(L^{4}(\Omega)\right)^{2}$, we have $K \cdot \nabla\left(H_{*}+\Theta\right) \in L^{4 / 3}(\Omega)$. Then, from the existence and uniqueness theorems for the Dirichlet problem for strong solutions and since the application

$$
(\Psi, u) \in\left(H_{0}^{1}(\Omega)\right)^{2} \longrightarrow(\nabla \Psi, \nabla u)
$$

is continuous and coercive, we deduce that $\Psi^{*} \in W^{2,(4 / 3)}(\Omega)$. Thus, $\nabla \Psi_{*} \in\left(W^{1,(4 / 3)}(\Omega)\right)^{2}$ in which $W^{1,(4 / 3)}(\Omega) \rightarrow L^{4}(\Omega)$ (see [11], Theorem 2.72), that is, $\Psi_{*} \in\left(W^{1,4}(\Omega)\right)^{2}$. Then, from Proposition 1, there exists a unique $H \in H^{2}(\Omega) \cap H_{0}^{1}(\Omega)$ satisfying $\left\{\begin{array}{l}\lambda(\nabla H, \nabla v)-\left(H \nabla v, \nabla \Psi_{*}^{\perp}\right)=\left(v \nabla \Theta, \nabla \Psi_{*}^{\perp}\right), \quad \forall v \in H_{0}^{1}(\Omega), \\ \lambda \Delta H-\nabla H \nabla \Psi_{*}^{\perp}=\nabla \Theta \nabla \Psi_{*}^{\perp} .\end{array}\right.$

On the other hand, from Remark 1, the bilinear form

$$
(H, v) \in\left(H_{0}^{1}(\Omega)\right)^{2} \longrightarrow(\nabla H, \nabla v)-\left(H \nabla v, \nabla \Psi_{*}^{\perp}\right)
$$

is continuous and coercive. Thus, from the Lax-Milgram theorem, we deduce that $H=H_{*}$. Hence, $K \cdot \nabla H_{*} \in L^{2}(\Omega)$, that is, $K \cdot \nabla\left(H_{*}+\Theta\right) \in L^{2}(\Omega)$, and thereby, from the existence and uniqueness theorems for the Dirichlet problem for strong solutions ([12], Theorem 9.15, problem 9.8) and from the Lax-Milgram theorem, $\Psi_{*}$ is the unique function in $H^{2}(\Omega) \cap H_{0}^{1}(\Omega)$ obeying

$$
\left\{\begin{array}{l}
\left(\nabla \Psi_{*}, \nabla u\right)=-\left(K \cdot \nabla\left(H_{*}+\Theta\right), u\right), \quad \forall u \in H_{0}^{1}(\Omega), \\
\Delta \Psi_{*}=K \cdot \nabla\left(H_{*}+\Theta\right) .
\end{array}\right.
$$

By the same method as the one used in the previous proposition, we get

$$
(\Psi, H) \in\left(W^{2,4}(\Omega)\right)^{2} .
$$

This completes the proof.

Remark 5. Notice that in Step 3 of the previous proof, Proposition 1 is necessary. Also, we need Lemma 1 and Remark 1, particularly, $\left(H \nabla H, \nabla \Psi^{\perp}\right)=0$ for $H \in H_{0}^{1}(\Omega)$ and $\Psi \in W^{1,4}(\Omega)$ which is a different result from ([8], Lemma 2.1) and it is proved by the generalized Green formula.

In addition to the previous results, we will study the existence of solutions in $C^{n}(\Omega), n \geq 3$.

Theorem 2. Let us assume, in addition to the hypotheses of the previous theorem, that $\partial \Omega$ is of class $C^{n}$, $T_{\omega} \in H^{n-(1 / 2)}(\partial \Omega)$, and $K \in W^{n-2,4}(\Omega)$ with $n \geq 2$. Then, problem (15)-(18) have at least one solution $(\Psi, H)$ in the space $H^{n}(\Omega) \times H^{n}(\Omega)$.

Proof. Let us assume $n \geq 3$, and let $(\Psi, H) \in\left(W^{2,4}(\Omega)\right)^{2}$ be a solution of problem (15)-(18). Then, we have

$$
\nabla \Psi, \nabla H \in\left(H^{1}(\Omega) \cap L^{\infty}(\Omega)\right)^{2} .
$$






Figure 1: Profiles of $f^{\prime}(\eta)$ for the case of prescribed heat at $\gamma=0.5$.

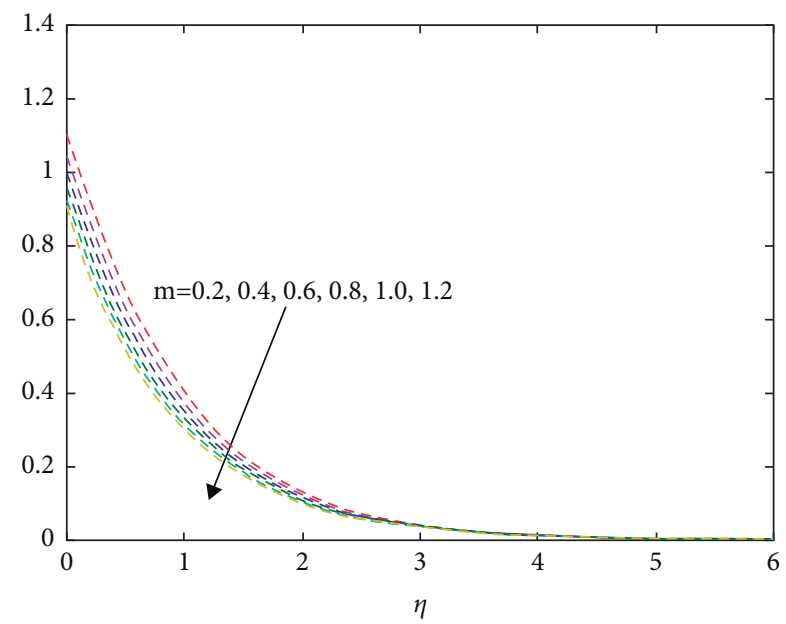

Figure 2: Profiles of $f^{\prime}(\eta)$ for the case of prescribed heat flux at $\gamma=0.5$.

TABle 1: Values of $f^{\prime \prime}(0)$ for the case of prescribed heat at $\gamma=0.5$.

\begin{tabular}{lc}
\hline $\mathrm{M}$ & $f^{\prime \prime}(0)$ \\
\hline 0.1 & -0.383276439 \\
0.2 & -0.446235343 \\
0.3 & -0.501716988 \\
0.4 & -0.551568992 \\
0.5 & -0.597004528 \\
0.6 & -0.638866144 \\
\hline
\end{tabular}

TABLe 2: Values of $f^{\prime}(0)$ for the case of prescribed heat flux at $\gamma=0.5$.

\begin{tabular}{lc}
\hline$M$ & $f^{\prime}(0)$ \\
\hline 0.2 & 1.109186225 \\
0.4 & 1.047023594 \\
0.6 & 0.998565092 \\
0.8 & 0.959474627 \\
1.0 & 0.927113207 \\
1.2 & 0.899773212 \\
\hline
\end{tabular}

On the other hand, from (12) and by the regularity theorem of elliptic problem ([12], Theorem 8.13), $\Theta$ is in $H^{n}(\Omega)$. It then follows that

$$
\partial_{x_{i}} \Theta \in H^{n-1}(\Omega) \cap L^{\infty}(\Omega) .
$$

Since $K \in\left(W^{n-2,4}(\Omega)\right)^{2}, n \geq 3$, we have

$$
\begin{aligned}
& K \cdot \nabla \Theta \in H^{1}(\Omega), \\
& K \cdot \nabla H \in H^{1}(\Omega) .
\end{aligned}
$$

From the regularity theorem of elliptic problem ([12], Theorem 8.13), we deduce that $\Psi$ is in $H^{3}(\Omega)$. Hence,

$$
\begin{gathered}
\nabla \Theta \cdot \nabla \Psi^{\perp}, \\
\nabla H \cdot \nabla \Psi^{\perp} \in H^{1}(\Omega),
\end{gathered}
$$

that is, $H \in H^{3}(\Omega)$. Continuing the above process, we obtain, after $n-2$ steps, $(\Psi, H) \in\left(H^{n}(\Omega)\right)^{2}$.

Let us denote by $\gamma_{0}$ the trace operator on $\partial \Omega$. Then, we have the following result.

Corollary 1. Let us assume, in addition to the hypotheses of the previous theorem, that $\partial \Omega$ is of class $C^{\infty}$ and $K \in\left(C^{\infty}(\bar{\Omega})\right)^{2}$ and there exists $\varphi \in C^{\infty}(\bar{\Omega})$ such that $\gamma_{0}(\varphi)=T_{\omega}$. Then, problem (15)-(18) have at least one solution $(\Psi, H)$ in the space $C^{\infty}(\bar{\Omega}) \times C^{\infty}(\bar{\Omega})$.

Remark 6. If $\Gamma_{1}$ and $\Gamma_{2}$ are closed and open subsets and $\Omega$ is sufficiently smooth (being able to apply Poincaré's inequality), then by applying Theorem 2.24 given in ([15], p. $132)$ or Theorem 5.8 given in ([16], p. 146), we can prove that the previous results of the existence of strong solutions are still valid in the general case where $\Gamma_{2} \neq \varnothing$.

\section{Conclusion}

In this paper, we study a problem given by two strongly coupled partial differential equations in a two-dimensional bounded domain, modeling a phenomenon of convective flow created by a heated and diving plate in a porous medium saturated with a fluid. We have established the existence of strong solutions without assuming that $\|\Psi\|_{H^{2}}$ is bounded by a certain constant (nor $\|K \nabla \Theta\|_{2}$ is small enough) and by choosing $K$ in $W_{2}^{4}$ (div, $\Omega$ ). Besides, we have added a new result (which does not exist in the literature) which is the existence of solutions of class $C^{n}, n \geq 3$ (particularly, solutions of class $C^{\infty}$ ).

\section{Appendix}

\section{Numerical Example}

Let us consider a semi-infinite vertical permeable or impermeable flat plate embedded in a fluid-saturated porous medium at the ambient temperature $T_{b}$. The rectangular Cartesian co-ordinates system is applied with the origin fixed at the leading edge of the vertical plate. The $x$-axis is directed upward along the plate, and the $y$-axis normal to it. 
It is assumed that the porous medium is homogeneous and isotropic where all the properties of the fluid and the porous medium are constants except the density. Also, the flow is incompressible and follows the Darcy-Boussinesq law. Based on these assumptions, the following governing equations are introduced:

$$
\begin{aligned}
& \frac{\partial^{2} \Psi}{\partial x^{2}}+\frac{\partial^{2} \Psi}{\partial y^{2}}=\partial_{y} T \\
& \frac{\partial^{2} T}{\partial x^{2}}+\frac{\partial^{2} T}{\partial y^{2}}=\partial_{x} T \partial_{y} \Psi-\partial_{y} T \partial_{x} \Psi
\end{aligned}
$$

with boundary conditions

$$
\begin{aligned}
\partial_{x} \Psi(x, 0) & =-\omega x^{(m-1) / 2}, \\
\partial_{x} T(x, 0) & =-x^{m}, \\
T(x, 0) & =T_{\omega}(x)=T_{b}+A x^{m}, \\
\partial_{y} \Psi(x, 0) & =x^{m}, \\
\partial_{y} \Psi(x, b) & =0, \\
T(x, b) & =T_{b},
\end{aligned}
$$

with $m \in \mathbb{R}, A, b>0, \lambda=1$ and $K \equiv(1,0)$. The parameter $\omega \in \mathbb{R}$ is the mass transfer coefficient where $\omega=0$ refers to the impermeable wall. Also, the permeable surface is represented by $\omega<0$ for the fluid suction and $\omega>0$ for the fluid injection.

Now, a brief discussion for the similarity solutions is given. Assuming that the convection takes place in a thin layer around the plate (we can neglect $\partial^{2} \Psi / \partial x^{2}$ and $\left.\partial^{2} T / \partial y^{2}\right)$ and hence the boundary layer approximation is obtained:

$$
\begin{aligned}
& \frac{\partial^{2} \Psi}{\partial y^{2}}=\frac{\rho_{b} \beta \cdot g_{2}}{\mu} \partial_{y} T, \\
& \frac{\partial^{2} \Psi}{\partial y^{2}}=\partial_{x} T \partial_{y} \Psi-\partial_{y} T \partial_{x} \Psi,
\end{aligned}
$$

with same boundary conditions (A.3)-(A.4).

For the case of prescribed heat, we introduce the new dimensionless similarity variables

$$
\begin{aligned}
\eta & =\left(R a_{x}\right)^{1 / 2} \frac{y}{x} \\
\Psi(x, y) & =\left(R a_{x}\right)^{1 / 2} f(\eta), \\
T(x, y) & =\left(T_{\omega}(x)-T_{b}\right) \theta(\eta)+T_{b},
\end{aligned}
$$

where

$$
R a_{x}=\frac{\rho_{b} \beta \cdot g\left(T_{\omega}(x)-T_{b}\right) x}{\mu}
$$

is the local Rayleigh number; equations (A.1) and (A.2) with boundary conditions (A.3) and (A.4) lead to the third-order ordinary differential equation

$$
f^{\prime \prime \prime}+\frac{m+1}{2} f f^{\prime \prime}-m f^{\prime 2}=0
$$

on $[0, b)$ subjected to

$$
\begin{aligned}
f(0) & =-\gamma, \\
f^{\prime}(0) & =1, \\
f^{\prime}(b) & =0,
\end{aligned}
$$

where

$$
\gamma=\frac{2 \omega}{m+1} \sqrt{\frac{\mu}{\rho_{b} \beta \cdot g \cdot A}} .
$$

In the case of prescribed heat flux, we introduce the new dimensionless similarity variables:

$$
\begin{aligned}
\eta & =3^{-(1 / 3)} R_{a}^{(1 / 3)} x^{(m-1) / 3} y, \\
\Psi(x, y) & =3^{2 / 3} R_{a}^{(1 / 3)} x^{(m+2) / 3} f(\eta) \\
T(x, y) & =3^{(1 / 3)} R_{a}^{-(1 / 3)} x^{(2 m+1) / 3} \theta(\eta)+T_{b}
\end{aligned}
$$

where the Rayleigh number is

$$
R_{a}=\frac{\rho_{b} \beta \cdot g}{\mu} .
$$

Equations (A.5) and (A.6) with boundary conditions (A.3) and (A.4) lead to the third-order ordinary differential equation

$$
f^{\prime \prime \prime}+(m+2) f f^{\prime \prime}-(2 m+1) f^{\prime 2}=0,
$$

on $[0, b)$ subjected to

$$
\begin{aligned}
f(0) & =-\gamma, \\
f^{\prime \prime}(0) & =-1, \\
f^{\prime}(b) & =0, \\
\gamma & =\frac{3^{1 / 3} R_{a}^{-(1 / 3)} \omega}{m+2} .
\end{aligned}
$$

In order to show the effectiveness of main results, numerical results are presented in this part and discussed. Here, the transformed equations governing the case of prescribed constant heat and the prescribed heat flux at $\gamma=0.5$ are solved, numerically, using the fourthorder Runge-Kutta method based on the shooting technique. The obtained data are presented in Figures 1 and 2 for $f^{\prime}(\eta)$, as well as values of $f^{\prime \prime}(0)$ and $f^{\prime}(0)$ are included in Tables 1 and 2. It is seen that a strong solution is existed and the behaviors of $f^{\prime}(\eta)$ for all values of $m$ are asymptotic. In addition, the growth in the power index $m$ has negative impacts on the values of $f^{\prime}(\eta), f^{\prime \prime}(0)$, and $f^{\prime}(0)$ due to the decrease in the temperature distributions at the surface and thickness of the thermal boundary layers. 


\section{Data Availability}

No external data were used to support the study in this paper.

\section{Conflicts of Interest}

The authors declare that they have no conflicts of interest.

\section{References}

[1] S. Akesbi, B. Brighi, and J. D. Hoernel, "Steady free convection in a bounded and saturated porous medium," in Proceedings of the 2004 Swiss-Japanese Seminar On Recent advances on Elliptic and Parabolic Issues, M. Chipot and H. Ninomiga, Eds., pp. 1-17, World Scientific Publishing Co. Pte. Ltd, Zurich, Switzerland, December 2004.

[2] M. Aiboudi, K. Boudjema Djeffal, and B. Brighi, "On the convex and convex-concave solutions of opposing mixed convection boundary layer flow in a porous medium," $A b$ stract and Applied Analysis, vol. 2018, Article ID 4340204, 5 pages, 2018.

[3] M. Aiboudi and B. Brighi, "On the solutions of a boundary value problem arising in free convection with prescribed heat flux," Archiv der Mathematik, vol. 93, no. 2, pp. 165-174, 2009.

[4] B. Brighi, A. Fruchard, and T. Sari, "On the Blasius problem," Advances in Differential Equations, vol. 13, pp. 509-600, 2008.

[5] G. C. Yang, "An upper bound on the critical value $\beta^{*}$ involved in the blasius problem," Journal of Inequalities and Applications, vol. 2010, Article ID 960365, 6 pages, 2010.

[6] A. Makhfi and R. Bebbouchi, "On the generalized Blasius equation," Afrika Matematika, vol. 31, no. 5-6, pp. 803-811, 2020.

[7] H. Aminikhah and S. Kazemi, "Numerical solution of the Blasius viscous flow problem by quartic $b$-spline method," International Journal of Engineering Mathematics, vol. 2016, Article ID 9014354, 6 pages, 2016.

[8] B. Brighi and S. Guesmia, "Existence of solutions and iterative approximations for nonlinear systems arising in free convection," Analysis and Applications, vol. 07, no. 03, pp. 225-241, 2009.

[9] B. Brighi and S. Guesmia, "On a nonlinear system of PDE's arising in free convection," Journal of Elliptic and Parabolic Equations, vol. 1, no. 2, pp. 263-269, 2015.

[10] H. Le Dret, Nonlinear Elliptic Partial Differential Equations, Springer International Publishing AG, Cham, Switzerland, 2018.

[11] F. Demengel and G. Demengel, Functional Spaces for the Theory of Elliptic Partial Differential Equations, SpringerVerlag, London, UK, 2012.

[12] D. Gilbarg and N. S. Trudinger, Elliptic Partial Differential Equations of Second Order, Springer-Verlag, Heidelberg, Germany, 1983.

[13] H. Brezis, Functional Analysis, Sobolev Spaces and Partial Differential Equations, Springer Science-Business Media, New York, NY, USA, 2011.

[14] V. Pata, Fixed Point Theorems and Applications, Springer Nature Switzerland AG, Cham, Switzerland, 2019.

[15] G. M. Troianiello, Elliptic Differential Equations and Obstacle Problems, Plenum Press, New York, NY, USA, 1987.

[16] J. Lopez-Gomez, Linear Second Order Elliptic Operators, World Scientific Publishing Co Pte Ltd, Singapore, 2013.
[17] B. Brighi, "The equation $f^{\prime \prime \prime}+f f^{\prime \prime}+g\left(f^{\prime}\right)=0$ and the associated boundary value problems," Results in Mathematics, vol. 61, no. 3-4, pp. 355-391, 2012.

[18] B. Brighi and S. Guesmia, "On a nonlinear system of PDE's arising in free convection," in Proceedings of the conference Recent Advences on Nonlinear Parabolic and Elliptic Differential Equations, pp. 35-43, Ryukoku University, Otsu, Japan, December 2007, https://hal.archivesouvertes. fr/hal00224397.

[19] L. C. Evans, Partial Differential Equations, American Mathematical Society, Boston, MA, USA, 1998.

[20] J. H. Merkin and G. Zhang, "On the similarity solutions for free convection in a saturated porous medium adjacent to impermeable horizontal surfaces," Wärme- und Stoffübertragung, vol. 25, no. 3, pp. 179-184, 1990.

[21] K. Taira, Analytic Semigroups and Semilinear Initial Boundary Value Problems, Cambridge University Press, London, UK, 2016.

[22] K. Taira, Boundary Value Problems and Markov Processes, Springer International Publishing, Heidelberg, Germany, 2020.

[23] K. Taira, Semigroups, Boundary Value Problems and Markov Processes, Springer-Verlag, Heidelberg, Germany, 2014.

[24] L. Tartar, An Introduction to Sobolev Spaces and Interpolation Spaces, Springer-Verlag, Heidelberg, Germany, 2019.

[25] R. A. Wooding, "Convection in a saturated porous medium at large Rayleigh number or Peclet number," Journal of Fluid Mechanics, vol. 15, no. 4, pp. 527-544, 1963. 